Research Article

\title{
The Visibility of Multisensor Fusion Technology in Public Art Design
}

\author{
Yan Zhang' and Qi Zhang $\mathbb{B D}^{2}$ \\ ${ }^{1}$ Jilin University of Architecture and Technology, Changchun, Jilin 130111, China \\ ${ }^{2}$ Changchun University of Technology, Changchun, Jilin 130012, China \\ Correspondence should be addressed to Qi Zhang; zhangq@ccut.edu.cn
}

Received 15 August 2021; Revised 16 October 2021; Accepted 9 November 2021; Published 20 December 2021

Academic Editor: Haibin Lv

Copyright (C) 2021 Yan Zhang and Qi Zhang. This is an open access article distributed under the Creative Commons Attribution License, which permits unrestricted use, distribution, and reproduction in any medium, provided the original work is properly cited.

\begin{abstract}
This exploration is aimed at improving the efficiency and safety of fully automatic line operation in public space. From the perspective of multisensor fusion technology, the definition, development status, and design principles of public art, as well as the related applications of multisensor fusion technology theory and image fusion theory, are introduced by consulting relevant literature. Then, the scheme of subway platform door gap antipinch detection system based on multisensor fusion and the obstacle detection method of tram in transit are proposed. Finally, through the method of questionnaire, the passengers' cognition of public art in subway space and the concerned components of public art in subway space are analyzed. The results show that the subway space art content loved by passengers is mainly daily life, and the degree of love can reach $28 \%$, followed by local customs, culture, and fashion trends, with a degree of love of $23 \%$. Besides, the problem of obstacle detection of tram in transit is also studied, and a new obstacle detection method combining visual sensor transmission detection and lidar detection is proposed. This method can quickly and accurately identify unsafe factors. Therefore, the research on the visibility of multisensor fusion technology in public art design has great reference significance for the rapid development of transportation industry.
\end{abstract}

\section{Introduction}

Public art design is of great significance in urban space. Public areas, commercial blocks, and subway spaces in the city need to be artistically rendered through decoration and design to publicize and spread urban civilization from the perspective of social culture [1]. However, people's pace of life is accelerating with the advancement of urbanization and their pursuit of culture and art is decreasing, which makes urban civilization no longer having characteristics and becoming a stereotyped scene. Hence, the art design of public areas must be paid enough attention. Subway travel accounts for an increasing proportion in people's daily life in the field of modern public transport. With status of domestic and international research subway space as an example, its visual design should not only meet people's aesthetic needs but also provide some indicating and guiding functions to create an open and comfortable space for pas- sengers. Applying multisensor fusion technology to optimize the gap detection between subway platform door and train door and ensure passenger safety is the main research purpose. Regarding the limitations of current gap detection technology and the higher safety requirements of fully automatic lines in the future, a scheme of antipinch detection system for the gap between subway platform door and train door based on multisensor data fusion is proposed [2, 3]. The exploration is aimed at quickly and accurately identifying unsafe factors and improving the visibility of multisensor fusion technology in public art design. Therefore, the focus is to put forward corresponding solutions and suggestions after studying the scheme. Meanwhile, the multisensor image fusion technology of tram is also the focus of current research.

To sum up, after understanding the current development status and relevant principles of public art, this exploration puts forward the scheme of antipinch detection system of 
subway platform door crack based on multisensor fusion, as well as the obstacle detection method of tram in transit. Then the questionnaire method is used to study and analyze the subway space art. According to the research results, a new obstacle detection method is proposed, combining visual sensor transmission detection and lidar detection. Therefore, this exploration is aimed at providing reference for further in-depth analysis of the visibility of multisensor fusion technology in public art design.

\section{Literature Review}

Li (2020) proposed that public art is a part of the construction of urban places, and its design and regeneration are very conducive to citizens' perception and positioning of the surrounding environment. It can be inferred that the current public art is no longer just a research in the field of art but has involved many aspects of urban development [4].

At present, relevant researchers have carried out corresponding research on multisensor fusion technology. For example, Das et al. [5] proposed a multisensor data fusion method based on Bayesian filtering theory and ant colony algorithm, which is the origin of filtering algorithms included in most filtering technologies such as the Kalman filter [6]. Lee et al. [7] proposed a decision-making method based on multisensor data fusion technology, using the strategy of multisource data fusion, in order to avoid vehicles touching pedestrians during driving. This method uses the vehicle automatic braking system to predict the time from vehicle braking to complete collision avoidance and predicts and estimates the reasonable and accurate time and reasonable stopping distance for a period of timely parking according to the relative speed of pedestrians and other vehicles on the road during the train [5]. Bagheri et al. [8] built a basic framework based on semiglobal matching method in the field of optical image presentation to study the threedimensional reconstruction of SAR optical image and proposed a multisensor three-dimensional reconstruction algorithm based on this research [7]. At present, there have been relevant studies on the gap detection device between subway platform door and train door and the obstacle detection technology along the way. However, there is still a lack of research work on the combination of these two technologies and multisensor image information fusion technology. This exploration is aimed at the subway gap detection device and obstacle detection technology based on that. Many of the above scholars and experts have studied and analyzed the multisensor fusion technology from different angles. However, they only cut in from a small angle and did not comprehensively and deeply explore the visibility of this technology in public art design. This exploration is to use the multisensor fusion technology to discuss from the passengers' cognition of public art and the components of public art they concerned. The degree of data research is more in-depth, and the scope is also broader. Finally, some new theories are put forward, which has a far-reaching impact on the visibility research of multisensor fusion technology in public art design.

\section{Application of Questionnaire Method in Multisensor Fusion Technology}

3.1. Development of Public Art. The development of public art is gradually mature in the process of urbanization. It is the product of the continuous development of human society, and also a crucial part of the process of urbanization. It not only promotes the progress of urban civilization but also helps to inherit urban culture. The foothold of public art lies in the historical heritage and era background of a city, and the development trend is mainly based on the aesthetics and needs of the public [8]. The coordinated development of people and cities can be realized through the art design of public places. Some scholars pointed out that while helping cities obtain a good space environment and life experience, public art is also constantly creating new cultures and trends. The forms of public art are diverse, so it creates a diversified urban civilization, enhances the inclusiveness of the city, improves public participation, and helps people develop a new urban image. Generally, the core of public art lies in the design of public space and public participation. Its main functions include the following: (1) providing an exhibition platform for urban civilization to help artists show their artistic attainments and pursuit through the design of space environment; (2) beautifying public space, optimizing and improving urban space through art design, and creating an urban environment with artistic atmosphere; and (3) improving public aesthetic taste. Public art is characterized by the synergy of popularization and artistry and deeply implements the principle that real art serves the public [9].

Public space is the main place for the implementation of public art and the place where public activities are most concentrated. The art design of public space needs to meet the aesthetic needs of the public, play a visual impact, and realize the special function of public space. For example, the public art design of subway space should not only consider beauty but also guide the orientation of passengers and even play the role of safety warning.

3.2. Design Principles of Public Art. The publicity, complexity, diversity, enthusiasm, openness, and place of public art give the uniqueness of public art design, thus contributing to the unique aspects of public art design. Art reflects the times, and the times produce art [10]. People's understanding of art is also deepening with the continuous progress and development of society, which is the characteristic of the times of public art. The public's aesthetic taste will change with the development of the times, and the continuous development of science and technology will bring people more materials and technologies to use. Hence, public art design should change with the development of the times, closely combine with the times, and create excellent works that advance with the times [11].

Subway space is the most crowded, functional, and interactive area in urban space. The space is relatively closed and the passenger flow is large, making the subway space relatively special and the design requirements of its public art also relatively strict. Among them, priority must be given 


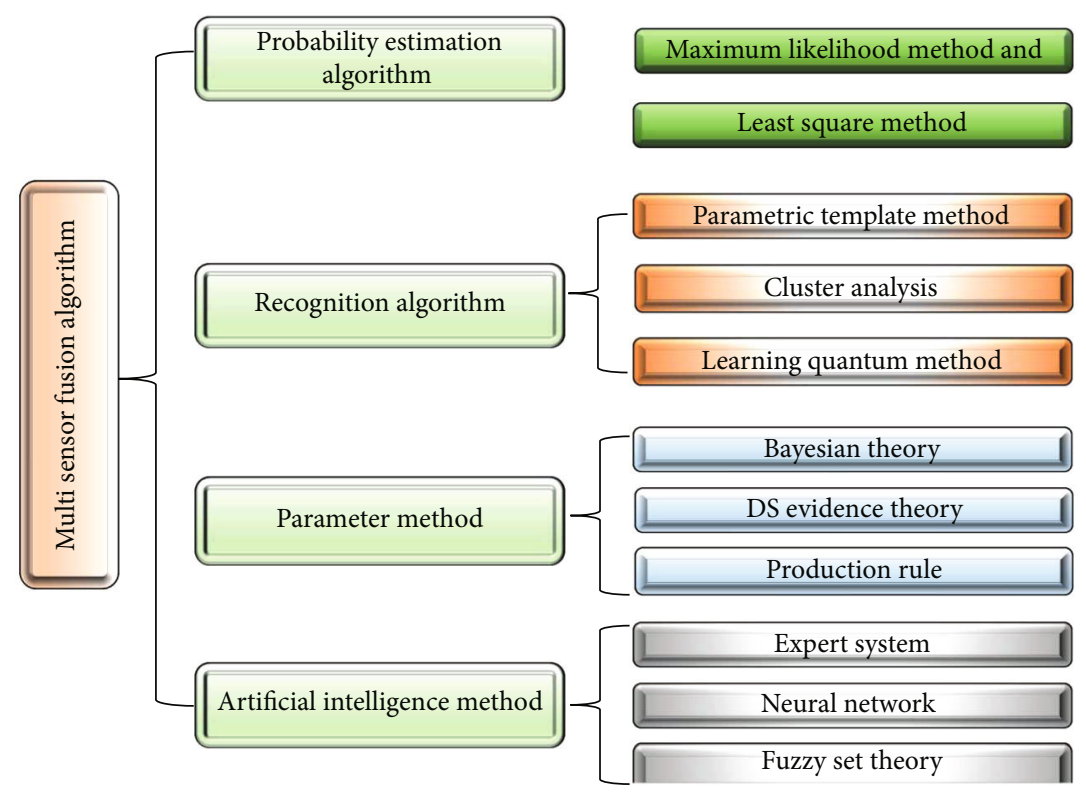

Figure 1: Classification diagram of the multisensor fusion algorithm.

to the principle of safety. There are huge potential safety hazards in the gap between the train and the platform door in the subway space, and passengers are very easy to be caught by the train door when getting on and off. Hence, the public art of the subway space needs to be designed according to the safety of passengers. How to perfectly integrate public art and subway space, educate and warn passengers' psychology through public art, and meet art needs is a problem worthy of in-depth study.

3.3. Multisensor Fusion Technology. The common methods of MSIF can be basically summarized into four categories, and each category of algorithm includes various typical methods, as shown in Figure 1.

MSIF is a comprehensive information processing process, which is more conducive to the implementation of subsequent decision-making tasks. Based on the sparse image block theory of the extended Kalman filter, the previous research focus in the field of MSIF also includes ROVIO [12]. Some classical model algorithms in MSIF, such as the well-known Kalman filter, hidden Markov algorithm, dynamic Bayesian network, and particle filter, are rooted in Bayesian filter [13]. Multisensor data fusion also has many advantages. Table 1 shows several methods of data fusion.

\subsection{Image Fusion and Recognition. By consulting massive} literature, the literature analysis method is adopted to briefly introduce the status of domestic and international research, the theory and application of multisensor fusion technology, and image fusion. Next, the scheme of subway platform door gap antipinch detection system based on multisensor fusion and the obstacle detection method of tram in transit are proposed. In the multisensor fusion decision-making proposed by some scientists, the methods of automatic detection of foreign objects can be roughly divided into two types: machine vision detection and physical detection [14]. Now- adays, image fusion has become a research hotspot, and image information fusion technology is a crucial and novel technology in the field of computer vision and image fusion and analysis [15]. Generally, for image fusion technology, multisource images in multisensor fusion technology can provide necessary complementary or redundant information for image information fusion. Image fusion technology can synthesize the effective information obtained from the comprehensive analysis of multisource images obtained from multiple sensors in the same scene. The antipinch detection system for the gap between subway platform door and train door based on multisensor fusion technology is formulated by selecting three types of sensors. The three types of sensors are laser radiation sensor, 3D laser scanning sensor, and image recognition sensor. Table 2 is the characteristic analysis table of the three sensors.

The MSIF gap antipinch detection system formed through the information fusion of the above three types of sensors can solve the shortcomings of the traditional single scheme and meet the needs of operation. If passengers or large objects are staying at the gap between platform door and train door, the image recognition system will send an alarm signal. Figure 2 presents its workflow.

In Figure 2, the positioning principle, characteristics, advantages, and disadvantages of four main internal sensors are introduced in detail. The four internal sensors are the visual camera sensor, lidar sensor, wheel gauge sensor, and IMU sensor. Among them, the visual camera sensor is the most widely used, and the lidar sensor is more accurate in some bad weather conditions [16]. The disadvantages of the above four internal sensors lead to that a single sensor cannot independently complete the positioning and navigation function in some poor weather conditions and bad environments. Thereby, several sensors can be used together to achieve accurate positioning and navigation, accurate identification and detection of obstacles in advance, and 
TABLE 1: Forms and advantages of data fusion.

Three forms of data fusion system

Time fusion

Space integration

A series
Characteristics of processing object

A series of measured values obtained from the same sensor, the same target or the same scene, but from different time, are fused.

Within a certain period of time, the measured values come from multiple same sensors with different spatial locations or from different sensors. This series of data is fused.
Advantages of multisensor data fusion

(i) The coverage of time and space has been expanded

(ii) The dimension and confidence of the measurement are increased

(iii) The detection performance is improved

(iv) Fault tolerance is excellent and the performance is more stable

(v) Spatial resolution is increased

(vi) The reliability and maintainability

of the system have been improved

(vii) The performance requirements

for a single sensor are reduced

TABLE 2: Characteristic analysis of three sensors.

\begin{tabular}{l} 
Sensor \\
$\begin{array}{r}\text { The technology is mature, and the detection distance is long. The fault is mainly the false alarm caused by the } \\
\text { alignment deviation of transmitting and receiving equipment caused by vibration. }\end{array}$ \\
$\begin{array}{r}\text { It can realize three-dimensional detection. Then, using its distance detection ability, it can realize the volume } \\
\text { identification and spatial position confirmation of foreign objects and make up for the blind area of laser } \\
\text { radiation detection. }\end{array}$ \\
$\begin{array}{r}\text { It can identify foreign objects and judge whether there are potential safety hazards. Besides, it uses its } \\
\text { visualization ability to assist managers in final confirmation and deal with faults efficiently. }\end{array}$ \\
\hline
\end{tabular}

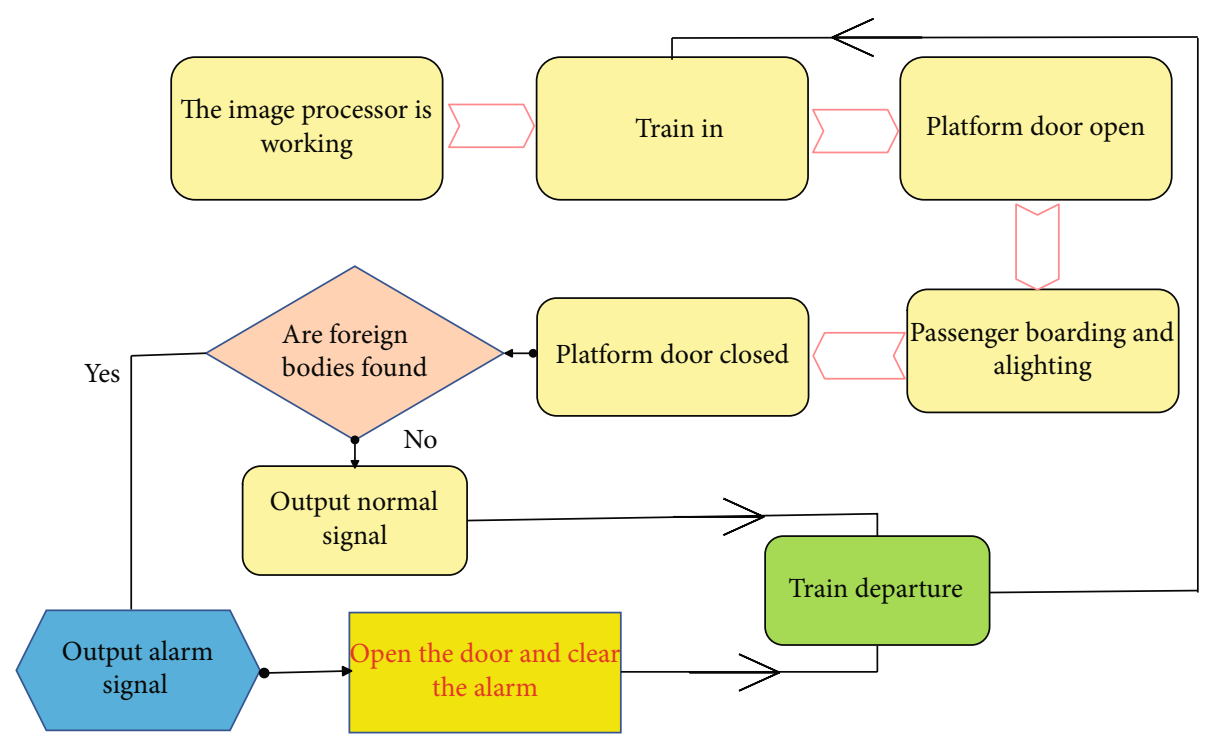

FIGURE 2: Workflow of image recognition system.

avoid traffic accidents. Multisensor data fusion not only expands the advantages of the fused sensors but also avoids some of their defects [17].

To sum up, vision camera sensor, lidar sensor, wheel gauge sensor, and IMU sensor are widely employed, and their disadvantages can be avoided by using them together. Multisensor fusion technology has played a great role in obstacle recognition, accurate navigation, and other issues. Besides, the vision camera sensor and lidar sensor comple- ment each other and work together, which can play the effect that one plus one is greater than two [18].

3.5. Front and Back Fusion Technology of Multisensor. The front fusion algorithm perceives the fused multidimensional comprehensive data, and there is only one perception. The front fusion algorithm collects and fuses the data information in the original layer. Fused data is like a super sensor, which can see not only infrared but also camera or RGB, 


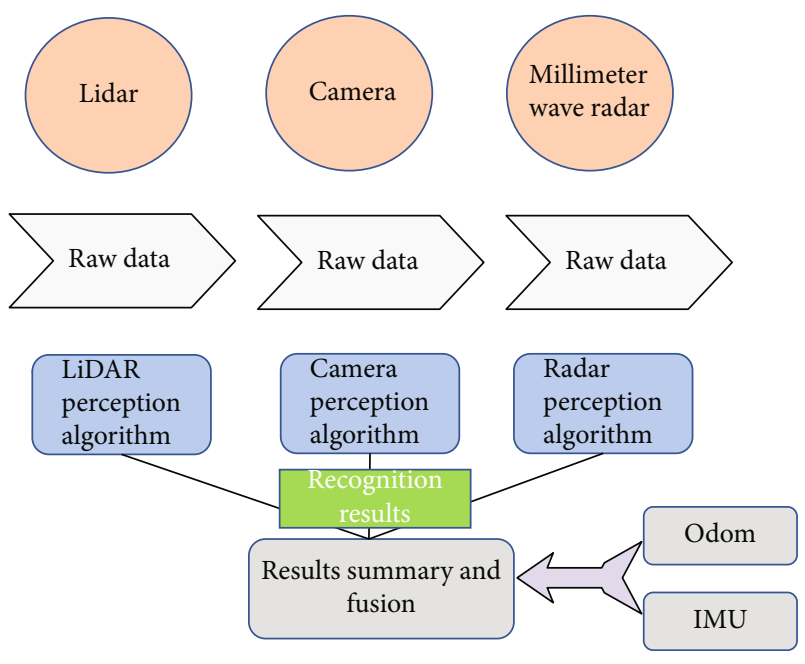

Figure 3: Typical structure of front fusion algorithm.

as well as lidar's three-dimensional information, just like a pair of super eyes [19]. On the super eye, the own perception algorithm is developed, and finally, an object in the result layer is output. Figure 3 shows a typical structure of the front fusion algorithm.

The postfusion algorithm requires each sensor applied in MSIF technology to process the generated target data independently of each other [20].

The postfusion algorithm mainly fuses the detection data of multiple detectors again and enables the same target detected by different detectors to do the postfusion again. In this way, it makes the confidence score of the target higher and the prediction frame more accurate, to achieve the postfusion. In the final analysis, the core of postfusion algorithm is to let the algorithm judge whether the detection results of multiple detectors are the same target. In the process of multidetector fusion, it needs to help the algorithm judge whether the detection of multiple detectors is the same object through clustering and find the corresponding same target detected by multiple detectors before similarity calculation. Prediction sample processing is as follows: after finding the same object detected by multiple detectors through clustering, this object is considered the prediction sample, and the similarity of the prediction samples is calculated to obtain the fusion results. Assume that all prediction frames related to candidate targets come from the same object. Therefore, the bounding box and confidence score of each prediction should be similar to each other. Based on this rule, the complete link clustering algorithm is used. Define the following similarity scoring functions for prediction clusters:

$$
\sigma\left(C_{i}, C_{j}\right)=\min \sigma(p, \widehat{p}) .
$$

In (1), $C_{i}$ and $C_{j}$ represent two different class groups, $\sigma$ is the confidence score, $p$ represents the prediction sample, and $\widehat{p}$ represents the transpose of the prediction sample. The termination conditions of class clustering are shown in the following equation.

$$
\max \sigma\left(C_{i}, C_{j}\right)<\tau
$$

In (2), $C_{i}$ and $C_{j}$ represent two different class groups, $\sigma$ is the confidence score, and $\tau$ indicates a super parameter. The similarity score of two prediction clusters associated with the same candidate target cannot be lower than this parameter.

Next, to predict the similarity between probabilities, it is assumed that several detections with great differences in category scores usually correspond to different prediction objects on the image. Combine the similarity of confidence scores into the similarity measurement, and use the Babbitt coefficient to measure the similarity between confidence scores:

$$
B C\left(\overline{c_{i}}, \overline{c_{j}}\right)=\sum_{K=1}^{K} \sqrt{\overline{c_{i}(k)} \overline{c_{j}(k)}} .
$$

In (3), $\bar{c}$ is obtained from the category score array $C$ by omitting the 0th element "no target," i.e., "background." $k$ represents the $k$ th prediction sample, and then, it is renormalized:

$$
\bar{c}^{(k)}=\frac{c^{(k)}}{1-c^{(0)}} .
$$

Two detections with similar shape, size, and position on the image often correspond to the same object. Consider the similarity between their bounding boxes to determine whether two detections correspond to the same object. Use the IOU coefficient to measure the similarity between bounding boxes.

$$
\begin{gathered}
\operatorname{IOU}\left(r_{i}, r_{j}\right)=\frac{r_{i} \cap r_{j}}{r_{i} \cup r_{j}}, \\
\sigma\left(p_{i}, p_{j}\right)=\operatorname{IOU}\left(r_{i}, r_{j}\right)^{\gamma} \times B C\left(\overline{c_{i}}, \overline{c_{j}}\right)^{1-\gamma} .
\end{gathered}
$$

$\gamma$ is a hyperparameter between 0 and 1 , when the prediction and confidence score of $p_{i}$ and $p_{j}$ are completely consistent; then $\sigma\left(p_{i}, p_{j}\right)$ is 1 . Before the postfusion step, each basic detector will eliminate the redundant bounding box. Hence, it is assumed that multiple similar detections from the same basic detector usually come from different objects. Therefore, the detection results from the same basic detector should be assigned to different class clusters. To achieve this, set the similarity score between the predictions of the same basic detector to 0 .

Each sensor has its own independent perception in the postfusion algorithm. For example, lidar has the perception of lidar, the camera has the perception of camera, and millimeter wave radar will make its own perception accordingly. After all sensors complete the target data generation, the data fusion is carried out by the main processor [21]. Figure 4 shows a typical structure of the postfusion algorithm.

3.6. Investigation and Analysis on the Correlation between Subway Space Public Art and Passengers' Cognition. In the public art design of subway space, the passengers' cognition and content preferences of public art need to be realized at 


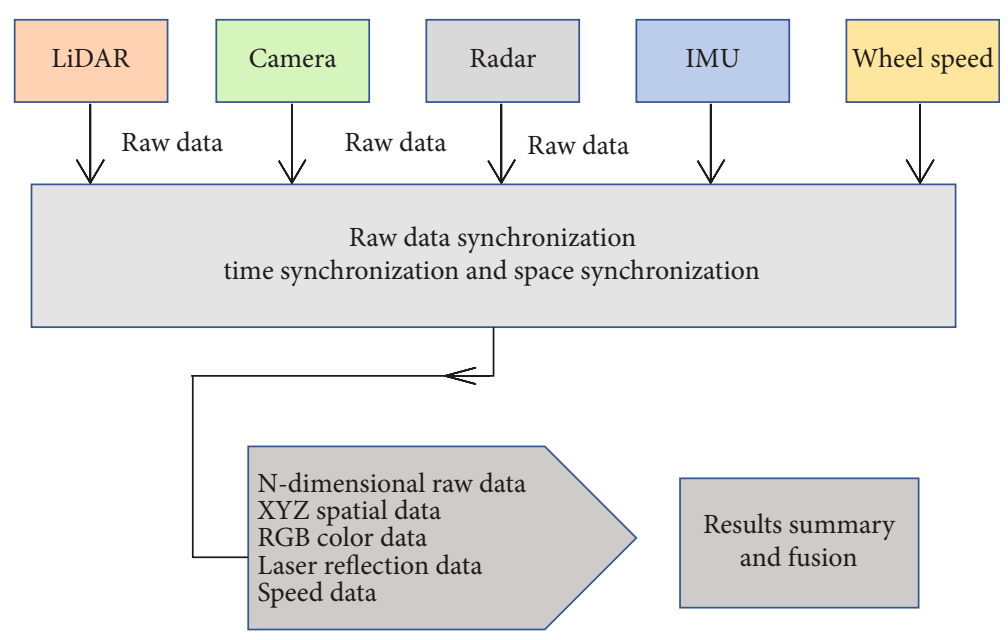

Figure 4: Typical structure of postfusion algorithm.

first. The most real passenger information in subway space can be obtained through field investigation under a certain base. As long as it is designed according to the acceptance and preferences of passengers, it can meet people's actual needs for public art. Hence, a public art cognition survey on different types of subway space passengers will be conducted in the form of questionnaire to deeply understand people's evaluation and attitude towards public art and carry out further public art design.

The content of the questionnaire is mainly evaluated and scored by the public art form of subway space, passengers' content preferences, and focus variables. The art forms mainly include murals, sculptures, wall decorative patterns, lighting and lighting systems, rest facilities decoration, LED billboards, column decoration, station music, and no advertising design in the carriage. The content preferences of passengers are mainly divided into nine aspects: daily life, natural scenery, local culture, historical stories, festival greetings, fashion trends, public welfare publicity, celebrity quotes, and others. The focus is divided into nine aspects: form, color, light and shadow, material, sound effect, humanization, regionality, participation, and recognition. Finally, the questionnaire is distributed to more than 30 cities across the country through the WeChat public platform, and a total of 147 valid questionnaires are recovered. Then, the questionnaire data are statistically analyzed by the SPSS25.0 software.

\section{Results and Discussion}

4.1. Passengers' Cognition of Public Art in Subway Space. Figure 5 displays the cognition of passengers in different fields on the public art forms of subway space according to the statistical results (Figures 5(a)-5(i), respectively, represent different art forms of murals, sculptures, wall decorative patterns, lighting and lighting systems, rest facilities decoration, LED billboards, column decoration, station music, and no advertising design in the carriage).

Figure 5 displays that in terms of public art forms of subway space, passengers in the art field are more sensitive to the cognition of public art, and their recognition of these art forms is relatively higher than that of passengers in other fields. Non art passengers have low recognition of these art forms. Generally, the recognition of music and LED billboards in the station is generally high, which can be generalized into the main form of public art in subway space. Then, the decoration of rest facilities and lighting system are also easy to be recognized. The most unrecognized art forms are mainly murals and sculptures, which are relatively abrupt in the subway space and have low acceptance.

Besides, Figure 6 shows the statistical results of passengers' content preferences about subway space art (where Figures 6(a)-6(i), respectively, represent daily life, natural scenery, local culture, historical stories, festival greetings, fashion trends, public welfare publicity, celebrity quotes, and others).

Figure 6 shows that the most popular content of subway space art is about daily life, followed by local culture and fashion trends, while public welfare publicity and festival greetings come last, indicating that most passengers prefer leisure and entertainment to too serious content.

\subsection{Principal Component Analysis of Subway Passengers'} Concerns about Public Art in Subway Space. In the questionnaire, there are mainly 9 variables set up according to the external attention of passengers to the public art of subway space, namely form, color, light and shadow, material, sound effect, humanization, regionality, participation, and recognition, which are expressed by $\mathrm{S} 1, \mathrm{~S} 2, \mathrm{~S} 3, \ldots, \mathrm{S} 9$, respectively. Figure 7 presents the final composition analysis results.

A questionnaire survey on passengers' concerns about public art in subway space is conducted. Figure 7 shows that among the nine variables, form, regionality, and recognition have strong correlation with the impact of the first external concern, while the variable humanization only has strong correlation with the impact of the second internal concern. It reveals that in terms of external concern, passengers focus on whether they can show regional characteristics in the form of public art and there is strong recognition; in terms of internal attention, passengers care about whether public art has humanized characteristics, whether it will affect their emotions and feelings, and so on. 


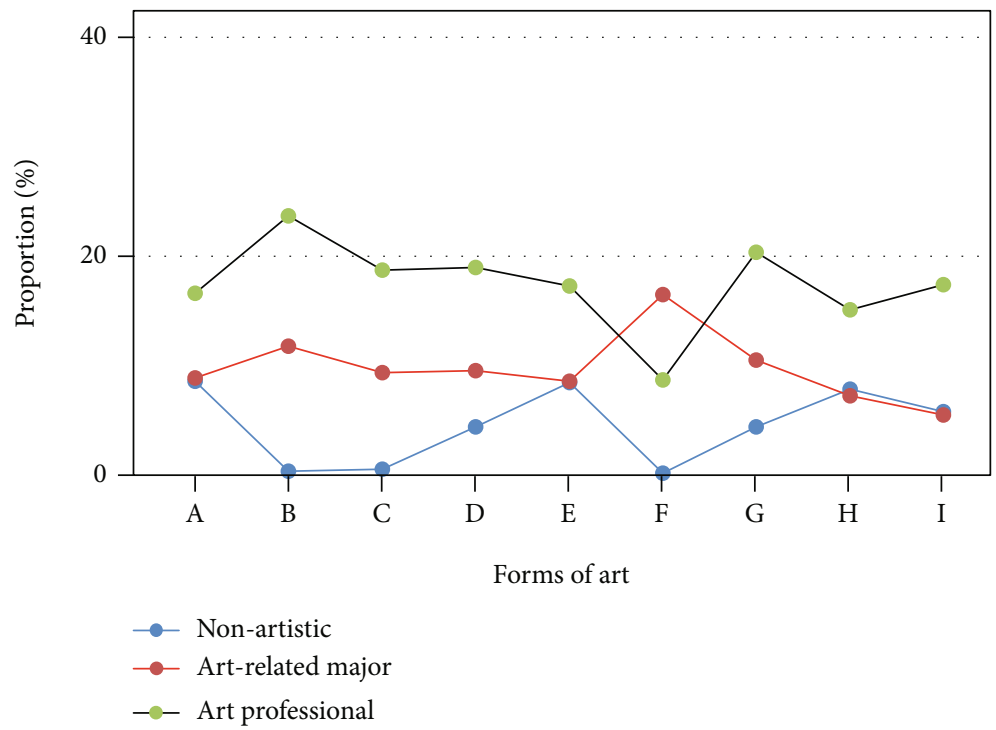

FIgURE 5: The cognition of passengers in different fields on the public art.

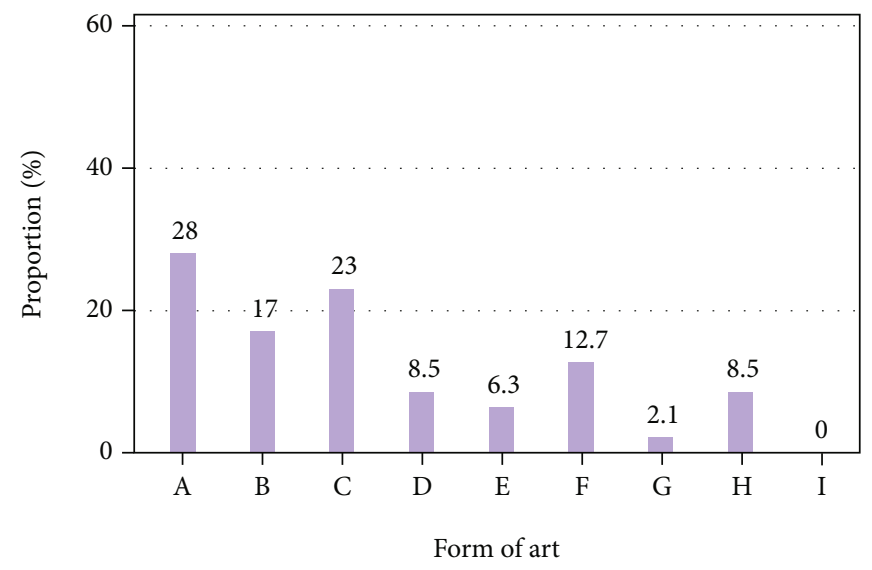

Figure 6: Public art content of subway space loved by passengers.

4.3. Research Results of Antipinch Detection System. Figure 8 displays the filtering results of the classical Kalman filter on real signals.

Figure 8 shows that overall, the input signal and the filtered signal fluctuate continuously. However, the test results of input signal and filtered signal will be smaller and smaller with the passage of time. It suggests that the classical Kalman filter has the function of real-time measurement of real signals. Moreover, the filter's amount of calculation will gradually decrease with the passage of time. On the one hand, it can save the time used in the calculation process and improve the work efficiency. On the other hand, it also makes the calculation results very accurate, and the filtering effect is better than other filters.

Multisensor data fusion (MSIF) is a new technology for the comprehensive processing of multisource data information from multiple sensors. As mentioned above, the Kalman filter is a classical filter in multisensor data fusion, which comes from the Bayesian filter. The classical Kalman filter has relatively good real-time performance, and the demand for calculation is very small. The filtering effect of the signal is also better than other filters.

The updating and upgrading of in transit obstacle detection technology are not only related to the driving safety of drivers but also an effective guarantee for the safety of pedestrians. Besides, the antipinch detection system studied is mainly based on MSIF technology, in which the classical data fusion technology in multisensor fusion technology is the Kalman filter. After the procedures of getting on and off passengers and closing the door after the subway stops, the antipinch system will send signals to three sensors: 3D laser scanning sensor, image sensor, and laser radiation sensor. Besides, image fusion technology begins to work. If two of the three decisions are abnormal, the locking circuit and train interlocking are disconnected. At this time, the train cannot leave the station, which can ensure the safety of passengers. 


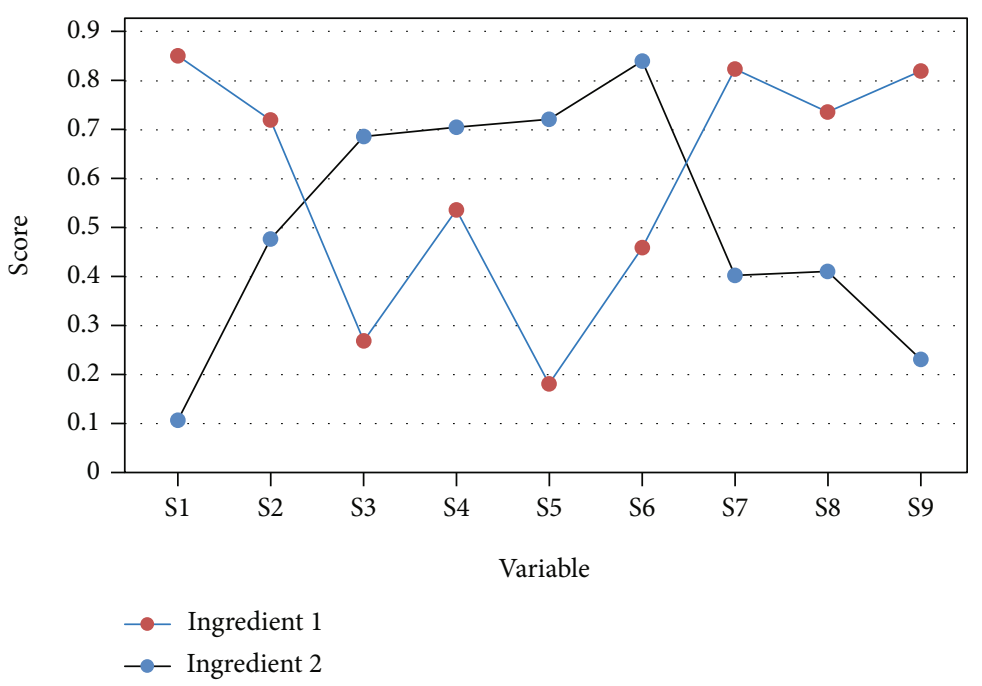

Figure 7: Principal component analysis diagram of concerns.

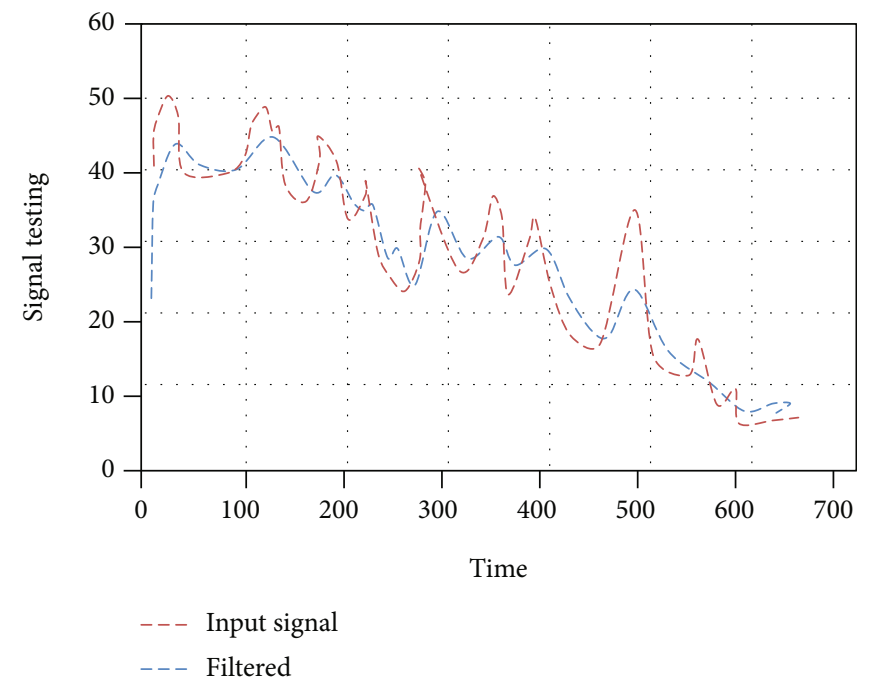

FIGURE 8: Filtering results of the classical Kalman filter on the real signal.

4.4. Analysis of Antipinch Detection System. Figure 9 is an antipinch detection system for the gap between subway platform door and train door designed based on multisensor fusion technology.

(1) When the subway stops at a station and completes the basic operation procedures such as getting on and off passengers and closing the platform door, the host of the gap antipinch detection system based on multisensor fusion sends the initial start trigger signal to the three sensors of 3D laser scanning sensor, image sensor, and laser radiation sensor, respectively; then, the image sensor can automatically start the image recognition work to intelligently identify the closing status of subway platform door and subway train door and whether there are abnormal events

(2) 3D laser scanning sensor, image sensor, and laser radiation sensor collect signals from the detection area and provide the collected information to the door head processing unit. The controller processes the collected signals of the three front-end sensors and provides the detected foreign matter result information to the "two out of three" decision-making device

(3) The "two out of three" decision-making device finally judges whether the train door is clamped with people and objects according to the results provided by the front-end 3D laser scanning sensor, image sensor, and laser reflector. If there are more than two schemes to judge the abnormal phenomenon of clamping people and objects at the platform train door as existence, the whole MSIF gap antipin detection system finally judges the abnormal phenomenon of clamping people and objects as existence. Moreover, the image sensor transmits the image and video information of people and objects to the 


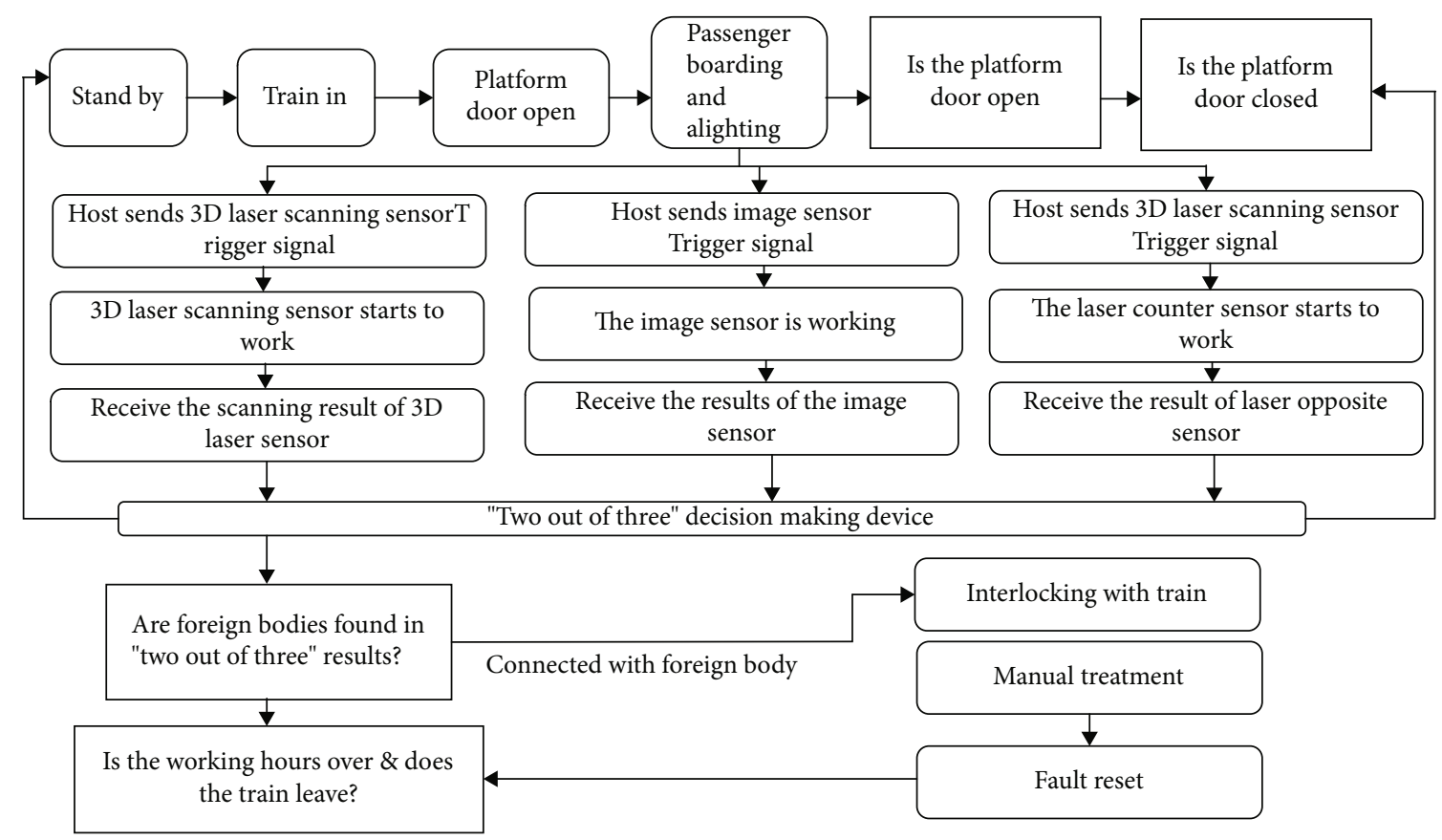

FIGURE 9: Detection process of multisensor fusion gap antipinch detection system.

display terminal for managers to check and confirm. If there is only one scheme to judge the abnormal situation, the system will judge that there is no abnormality. Then, the image sensor shall also record and store the image and video information in the alarm area for later false alarm analysis and confirmation

(4) If the MSIF gap antipinch detection system makes a "two out of three" decision judgment, and more than two schemes judge that the phenomenon of clamping people and objects at the platform train door exists, it is determined that the phenomenon of clamping people and objects exists. At this time, the system will automatically disconnect the locking circuit and interlock with the train, and the train cannot leave the station, so as to ensure the safety of passengers. Then, the system sends the alarm information of people and objects to the display terminal and mobile terminal on the platform for the station management personnel to quickly check and confirm whether there are people and objects and deal with them in time

(5) When the MSIF gap antipinch detection system makes a decision of "two out of three" and determines that there is the phenomenon of people and objects pinched in the train door, the system will send the alarm information to the display terminal and wait for the station management personnel to check and confirm. The system can be reset only after the abnormal conditions are manually confirmed and the phenomenon of people and objects clamped on the train door is handled
(6) When the abnormal state processing is completed and the subway is still in the station and within the specified detection time, the system will redetect the detection area until the detection time is over. In addition, the detection time specified by the system can be adjusted

(7) If the phenomenon that people and objects are clamped in the subway train door is not detected within the working time range of the system, the train can leave the station. At this time, the system will be in standby mode again, waiting for the next subway to arrive for detection. Grisetti (2018) studied the 2D laser system. Based on the particle filter algorithm, the amount of calculation is small, and the accuracy is high, which can be used for indoor real-time grid map construction. However, considering the number of particles and the calculated memory size, the algorithm is not suitable for large experimental environments [22]. This exploration is to analyze the visual design of public art from multiple angles by using multisensor fusion technology. Therefore, relatively speaking, the technologies and methods applied are more suitable for public art.

\section{Conclusion}

The multisensor fusion technology is adopted to study the art design of subway space, and the following conclusions are drawn. (1) The passengers' cognition of public art in subway space is studied through the method of questionnaire. It is found that passengers generally have high awareness of music and LED billboards. 
Then, the decoration of rest facilities and lighting systems can also be easily identified. The most unrecognized art forms are mainly murals and sculptures; (2) as for the main components of passengers' attention to public art in subway space, it is found that passengers' external attention is mainly form, regionality, and recognition, and passengers focus more on whether public art has humanized characteristics in their internal attention; (3) the problem of obstacle detection of tram in transit is also studied, and a new obstacle detection method combining the obstacle detection results transmitted by visual sensor with lidar detection results is proposed, which can quickly and accurately identify the unsafe factors; (4) the multisensor fusion gap antipinch detection system can quickly identify whether people or objects are clamped in the subway door within a limited time and can effectively improve the safety of passengers taking the subway.

The development of MSIF technology is in its infancy. The research of the above MSIF technology and image recognition in subway gap antipinch detection and the technology of obstacle detection along the way have been continuously improved, and new technologies have been introduced to combine with it. There is still much work to be done in the field of MSIF. The main research directions of MSIF in the future are as follows, such as the combination of MSIF technology and target recognition, the research of MSIF technology and image fusion, and the research of multisensor distributed detection.

The questionnaire method is employed to analyze and study the visibility of multisensor technology in public art design from multiple angles and finally draw the corresponding conclusions. These conclusions and viewpoints are brand-new, which can be used for reference for subsequent related research. Due to the limited energy, there are still some limitations in data acquisition, resulting in some deviations in the analysis of relevant data, and the economic investment of multisensor technology in public art design has not been discussed. Subsequently, the benefit evaluation can be carried out according to the specific situation, so that the technology can make a certain contribution to the visibility design of public art in the future.

\section{Data Availability}

The data used to support the findings of this study are available from the corresponding author upon request.

\section{Conflicts of Interest}

The authors declare no conflicts of existing interest.

\section{References}

[1] H. Y. Lei and W. M. Liu, "Detection of foreign objects in subway outdoor platform based on K-means algorithm," Computer and Modernization, vol. 6, pp. 42-46, 2018.

[2] M. Rao, "Design of top mounted anti-pinch automatic detection system between platform screen door and train door," Urban Mass Transit, vol. 20, no. 5, pp. 143-146, 2017.
[3] R. Boukezzoula, D. Coquin, T. L. Nguyen, and S. Perrin, "Multi-sensor information fusion: combination of fuzzy systems and evidence theory approaches in color recognition for the NAO humanoid robot," Robotics and Autonomous Systems, vol. 100, pp. 302-316, 2018.

[4] E. H. Lee and T. L. Song, "Multi-sensor track-to-track fusion with target existence in cluttered environments," Iet Radar Sonar \& Navigation, vol. 11, no. 7, pp. 1108-1115, 2017.

[5] S. Das, S. Barani, S. Wagh, and S. S. Sonavane, "Extending lifetime of wireless sensor networks using multisensor data fusion," Sādhanā, vol. 42, no. 7, pp. 1083-1090, 2017.

[6] L. Li, "Urban public art design based on regional culture - a case study of Zigong," Frontiers in Economics and Management, vol. 1, no. 4, p. 31, 2020.

[7] H. K. Lee, S. G. Shin, and D. S. Kwon, "Design of emergency braking algorithm for pedestrian protection based on multisensor fusion," International Journal of Automotive Technology, vol. 18, no. 6, pp. 1067-1076, 2017.

[8] H. Bagheri, M. Schmitt, P. d'Angelo, and X. X. Zhu, "Exploring the applicability of semi-global matching for sar-optical stereogrammetry of urban scenes," The International Archives of the Photogrammetry, Remote Sensing and Spatial Information Sciences, vol. XLII-2, pp. 43-48, 2018.

[9] S. Subedi, Y. D. Zhang, M. G. Amin, and B. Himed, "CramerRao type bounds for sparsity-aware multi-sensor multi-target tracking," Signal Processing, vol. 145, pp. 68-77, 2018.

[10] S. Auer, I. Hornig, M. Schmitt, and P. Reinartz, "Simulationbased interpretation and alignment of high-resolution optical and SAR images," IEEE Journal of Selected Topics in Applied Earth Observations \& Remote Sensing, vol. 10, no. 11, pp. 4779-4793, 2017.

[11] K. Sun, K. Mohta, B. Pfrommer et al., "Robust stereo visual inertial odometry for fast autonomous flight," IEEE Robotics and Automation Letters, vol. 3, no. 2, pp. 965-972, 2018.

[12] M. Bloesch, M. Burri, H. Sommer, R. Siegwart, and M. Hutter, "The two-state implicit filter recursive estimation for mobile robots," IEEE Robotics and Automation Letters, vol. 3, no. 1, pp. 573-580, 2018.

[13] B. Michael, O. Sammy, H. Marco, and S. Roland, "Robust visual inertial odometry using a direct EKF-based approach," IEEE/RSJ International Conference on Intelligent Robots and Systems (IROS), vol. 23, no. 4, pp. 298-304, 2015.

[14] Q. Xiao, Y. Zhao, and W. Huan, "Multi-sensor data fusion for sign language recognition based on dynamic Bayesian network and convolutional neural network," Multimedia Tools and Applications, vol. 78, no. 11, pp. 15335-15352, 2019.

[15] E. Taghavi, R. Tharmarasa, T. Kirubarajan, Y. Bar-Shalom, and M. Mcdonald, "A practical bias estimation algorithm for multisensor-multitarget tracking," IEEE Transactions on Aerospace and Electronic Systems, vol. 52, no. 1, pp. 2-19, 2016.

[16] W. P. Yu, J. Z. Zhang, J. D. Xu, and Y. W. Xu, “An accurate indoor map matching algorithm based on activity detection and crowdsourced Wi-Fi," Science China (Technological Sciences), vol. 62, no. 9, pp. 1492-1501, 2019.

[17] R. Mur-Artal and J. D. Tardós, “Orb-slam2: an open-source slam system for monocular, stereo, and rgb-d cameras," IEEE Transactions on Robotics, vol. 33, no. 5, pp. 1255-1262, 2017.

[18] J. Zhang and S. Singh, "Low-drift and real-time lidar odometry and mapping," Autonomous Robots, vol. 41, no. 2, pp. 401416, 2017. 
[19] M. Ramezani and K. Khoshelham, "Vehicle positioning in GNSS-deprived urban areas by stereo visual-inertial odometry," IEEE Transactions on Intelligent Vehicles, vol. 3, no. 2, pp. 208-217, 2018.

[20] T. Qin, P. Li, and S. Shen, "Vins-mono: a robust and versatile monocular visual-inertial state estimator," IEEE Transactions on Robotics, vol. 34, no. 4, pp. 1004-1020, 2018.

[21] Z. Lee and T. Q. Nguyen, "Multi-resolution disparity processing and fusion for large highresolution stereo image," IEEE Transactions on Multimedia, vol. 17, no. 6, pp. 792-803, 2015.

[22] S. Grisetti, "Bandwidth-constrained decentralized detection of an unknown vector signal via multisensor fusion," IEEE Transactions on Signal and Information Processing Over Networks, vol. 34, no. 4, pp. 104-112, 2018. 